\section{Rôle inattendu de l'arginine vasopressine comme inhibiteur de la réponse immunitaire}

Cécilia Chassin, Alain Vandewalle
Inserm U773, Centre de Recherche

Biomédicale Bichat-Beaujon (CRB3),

Université Paris 7-Denis Diderot,

Faculté de Médecine Xavier Bichat, BP 416,

16, rue Henri Huchard,

75870 Paris Cedex 18, France.

cecilia.chassin@bichat.inserm.fr

la dégradation de IKB- $\alpha$, la phosphorylation et l'activation du facteur de transcription NF-KB et la sécrétion de cytokines pro-inflammatoires (Figure 1). Des études antérieures ont montré que la dDAVP active la PP2A dans les cellules du tubule collecteur rénal [7]. La PP2A, qui est couplée au canal chlorure CFTR [8] est, de par son activité phosphatase, impliquée dans la régulation de nombreuses voies de signalisation [9]. Il est apparu que des inhibiteurs de la PP2A (calyculine $A$ et acide okadaïque) antagonisent l'action inhibitrice de la dDAVP sur la réponse inflammatoire. Le fait marquant est que des inhibiteurs de CFTR (tel que CFTR $_{\text {inh }}$-172, l'inhibiteur le plus spécifique de (FTR) ou l'invalidation de CFTR, antagonisent l'action inhibitrice de la dDAVP sur l'activation cellulaire induite par le LPS. Ces résultats ont permis de démontrer que la dDAVP inhibe la réponse inflammatoire des cellules du tubule collecteur induite par le LPS par un mécanisme faisant intervenir la PP2A et un canal CFTR fonctionnel.

\section{La dDAVP inhibe la réponse immunitaire dépendante de TLR4}

Afin de tester si ce mécanisme inhibiteur de la dDVAP peut intervenir in vivo, les effets de la dDAVP ont été analysés chez la souris dans un modèle expérimental d'infection du tractus urinaire réalisé par inoculation dans la vessie (par voie transurétrale) des $\varepsilon$. coli uropathogènes [1] (Figure 2). Des minipompes, délivrant de la dDAVP en 
continu, ont été insérées sous la peau de souris sauvages $\left(L p s^{n}\right)$ et de souris déficientes en TLR4 $\left(L p s^{d}\right)$, qui ont été ensuite inoculées avec une souche d'E. coli uropathogènes. L'administration chronique de dDAVP à des souris sauvages a induit une diminution significative de l'expression des ARNm des médiateurs pro-inflammatoires, de la sécrétion de cytokines pro-inflammatoires ainsi que de l'influx de polynucléaires neutrophiles ( $P N)$, et une augmentation concomitante de la charge bactérienne intrarénale par rapport aux souris non traitées. En revanche, l'infusion de dDAVP n'a eu aucun effet sur les souris déficientes en TLR4, démontrant que l'action inhibitrice de la dDAVP agit spécifiquement sur la voie de signalisation de TLR4 [11, 12].

Nous avons ensuite testé si le blocage in vivo du RV2 pouvait prévenir l'inhibition qu'exerce la dDAVP sur la réponse inflammatoire induite par les bactéries. À l'inverse des effets qu'entraîne l'administration de dDAVP, un prétraitement - avant leur infection avec une souche d'E. coli uropathogènes - des souris sauvages avec le SR121463B, un antagoniste du RV2 appartenant à la famille des Vaptans et préconisé dans le traitement des hyponatrémies réfractaires, a induit l'augmentation de l'expression des ARNm de médiateurs pro-inflammatoires, de la sécrétion des cytokines et de l'influx de PN, et une

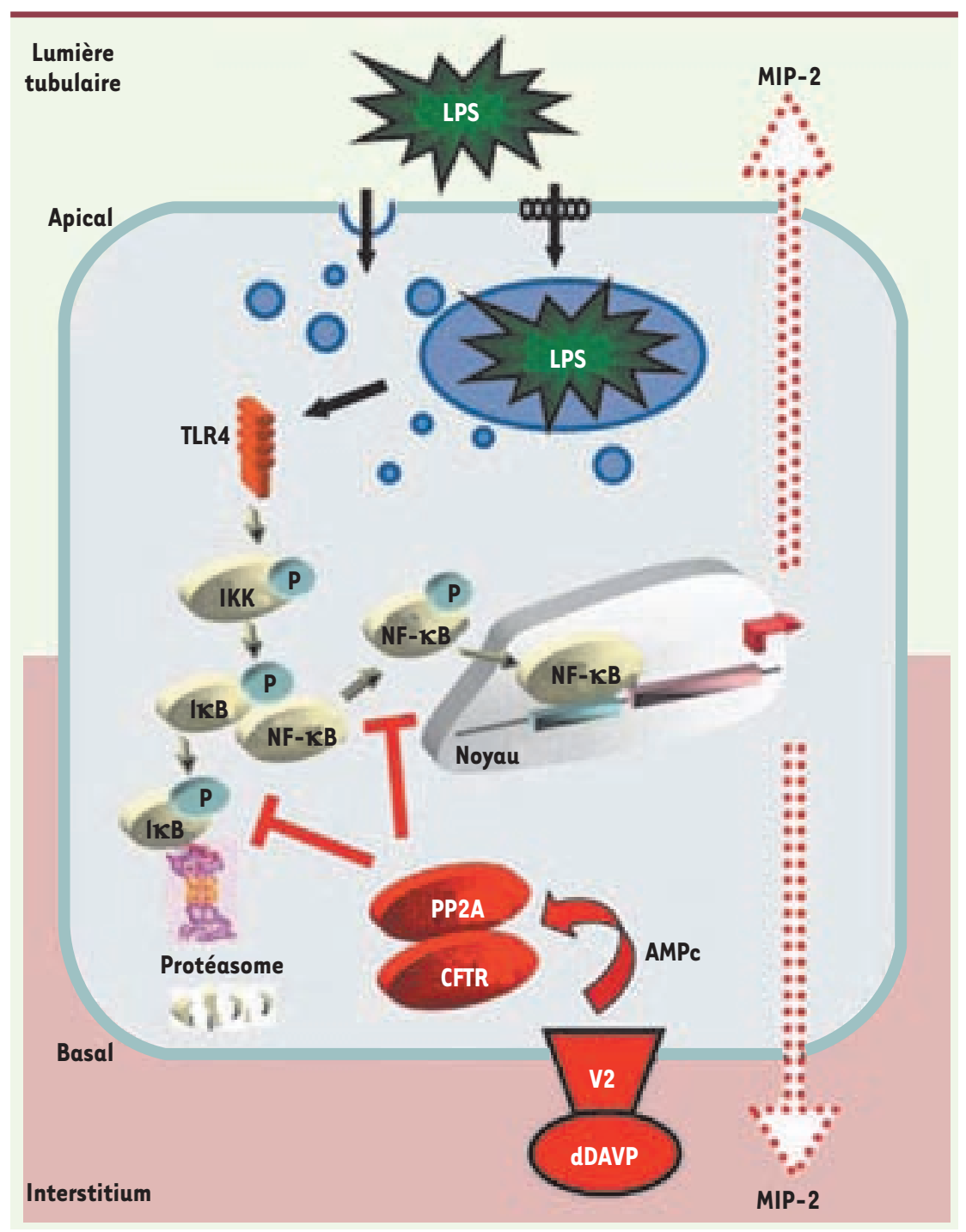

Figure 1. Mécanisme d'inhibition par la dDAVP de la réponse inflammatoire dépendante de TLR4 dans les cellules du tubule collecteur rénal. La reconnaissance du LPS des $\varepsilon$. coli uropathogènes par TLR4 exprimé dans les cellules du tube collecteur induit une cascade de signalisation responsable de la dégradation de $\mid \kappa B$ dans le protéasome et de la translocation nucléaire du facteur de transcription NF$\kappa B$ conduisant à la sécrétion bidirectionnelle de cytokines, tel que MIP-2. La dDAVP, dont la production est augmentée dans les états de déshydratation, se fixe à son récepteur V2 présent dans les membranes basolatérales de cellules du tube collecteur. L'activation de la protéine phosphatase 2A (PP2A) couplée au canal chlorure CFTR va inhiber la dégradation $d^{\prime} \mid \kappa B$ et la translocation de NF- $\kappa B$, ayant pour effet de diminuer la production de médiateurs de l'inflammation. 


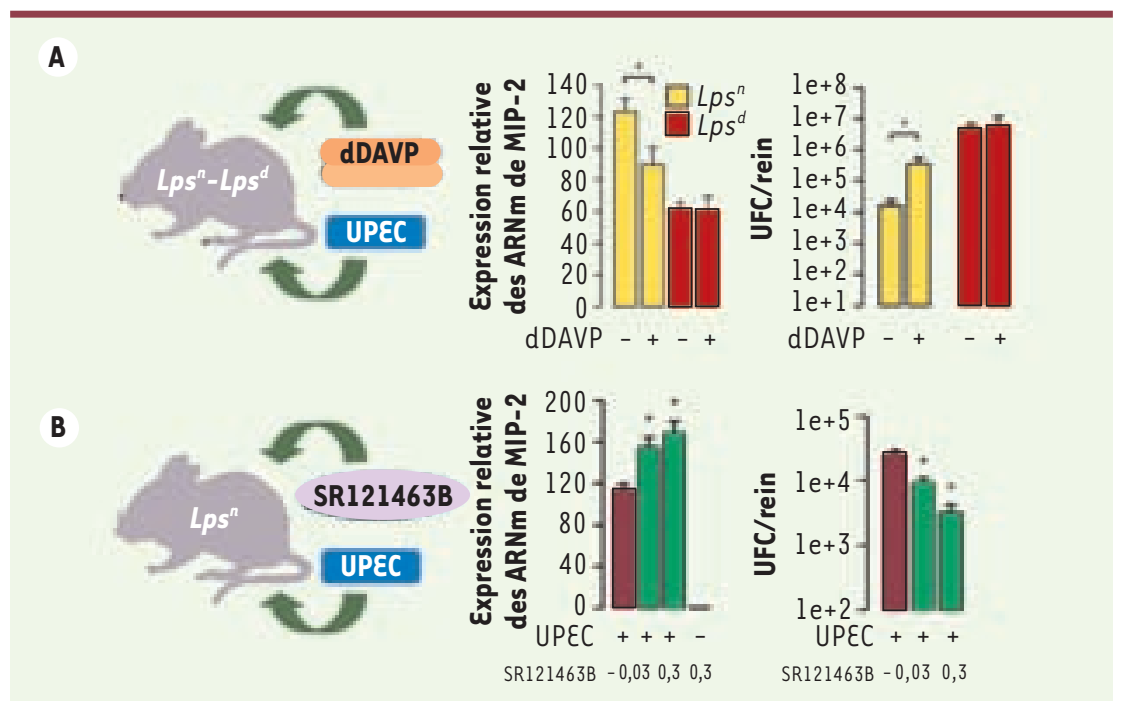

Figure 2. Mécanismes d'action de la dDAVP. La dDAVP inhibe la réponse inflammatoire dépendante de TLR4 induite par les $\varepsilon$. coli uropathogènes (UPEC) et favorise la colonisation des reins dans un modèle d'infection ascendante du tractus urinaire chez la souris. $A$. Des souris normales $\left(L p s^{n}\right)$ ou déficientes en TLR4 $\left(L p s^{d}\right)$ ont été inoculées par voie transurétrale avec des UPEC ( $10^{8}$ bactéries) 48 heures après l'implantation sous-cutanée de minipompes osmotiques délivrant de la dDAVP en continu ( $1 \mathrm{ng} / \mu \mathrm{l} / \mathrm{h})$. Vingt-quatre heures après l'infection, les souris ont été sacrifiées et les analyses effectuées sur les reins ont montré une diminution de l'expression des ARNm des cytokines pro-inflammatoires comme MIP-2 (macrophage inhibitory protein), et une augmentation de la charge bactérienne dans les reins [exprimée en nombre d'unités formant colonie (UFC) par rein] des souris Lps $^{n}$, mais pas des souris $L p s^{d}$, démontrant ainsi que la dDAVP inhibe la réponse inflammatoire dépendante de TLR4. B. A contrario, les analyses réalisées sur des reins de souris $L p s^{n}$ prétraitées avec un antagoniste du récepteur de la dDAVP, le SR121463B ( 0,03 ou 0,3 mg/kg), puis inoculées avec des UPEC, ont montré une augmentation de l'expression des ARNm de MIP-2 et une dimunition significative de la charge bactérienne ( $p<0,05$ entre les groupes).

\section{RÉFÉRENCES}

1. Chassin C, Goujon JM, Darche S, et al. Renal collecting duct epithelial cells react to pyelonephritisassociated Escherichia coli by activating distinct TLR4-dependent and -independent inflammatory pathways. J Immunol 2006; 177 : 4773-84.

2. Chassin C, Goujon JM, Le Bouguénec C, et al. Une nouvelle fonction pour les cellules intercalaires du tubule collecteur rénal : la lutte contre les Escherichia coli uropathogènes. Med Sci (Paris) 2007 ; $23: 32$-4.

3. Bens M, Van Huyen JP, Cluzeaud F, et al. CFTR disruption impairs cAMP-dependent $\mathrm{Cl}^{-}$secretion in primary cultures of mouse cortical collecting ducts. Am J Physiol Renal Physiol 2001 ; 281 : F434-42.

4. Panettieri RA Jr, Lazaar AL, Puré $\varepsilon$, et al. Activation of cAMP-dependent pathways in human airway smooth muscle cells inhibits TNF-alpha-induced ICAM-1 and VCAM- 1 expression and T lymphocyte adhesion. J Immunol 1995 ; 154 : 2358-65.

5. Aronoff DM, Canetti C, Serezani CH, et al. Cutting edge: macrophage inhibition by cyclic AMP (cAMP): differential roles of protein kinase $A$ and exchange protein directly activated by cAMP-1. J Immunol 2005 ; 174 : 595-9.

6. Chassin C, Hornef MW, Bens M, et al. Hormonal control of the renal immune response and antibacterial host defense by arginine vasopressin. J Exp Med 2007 ; $204: 2837-52$.

7. Blot-Chabaud M, Coutry N, Laplace M, et al. Role of protein phosphatase in the regulation of $\mathrm{Na}^{+}-\mathrm{K}^{+}$-ATPase by vasopressin in the cortical collecting duct. J Membr Biol 1996 ; $153: 233-9$.
8. Thelin WR, Kesimer M, Tarran R, et al. The cystic fibrosis transmembrane conductance regulator is regulated by a direct interaction with the protein phosphatase 2A. J Biol Chem 2005 ; 280 : 41512-20.

9. Mumby MC, Walter G. Protein serine/threonine phosphatases: structure, regulation, and functions in cell growth. Physiol Rev 1993; 73: 673-99.

10. Knepper MA, Star RA. Vasopressin: friend or foe? Nat Med 2008 ; 14 : 14-6.

11. Delneste $Y$, Beauvillain C, Jeannin P. Immunité naturelle: structure et fonction des Toll-like receptors. Med Sci (Paris) $2007 ; 23: 67-73$.

12. Musette $P$, Auquit Auckbur I, Begon $\varepsilon$. Immunité innée: expression cutanée et fonction des récepteurs Tolllike. Med Sci (Paris) 2006 ; 22 : 149-52.

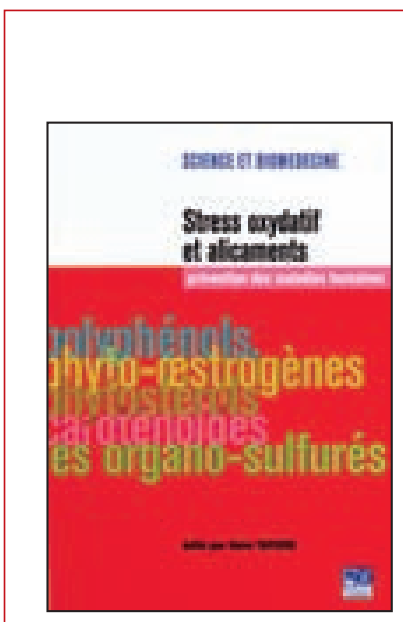

ISBN : 2-84254-111-1 86 pages

\section{Bon de commande}

À retourner à EDK, 2, rue Troyon - 92316 Sèvres Cedex

Tél. : 0155641393 - Fax : 0155641394 - E-mail : edk@edk.fr

NOM : Prénom :

Adresse :

Code postal :

Ville :

Pays :

Fonction :

Je souhaite recevoir l'ouvrage Stress oxydatif et alicaments : $14 €+3 €$ de port $=\mathbf{1 7} €$ TTC

en ................ exemplaire, soit un total de ...................................... €

$\square$ Par chèque, à l'ordre de $\mathbf{E} \mathbf{D} \mathbf{K}$

$\square$ Par carte bancaire : $\quad \square$ Visa $\square$ Eurocard/Mastercard

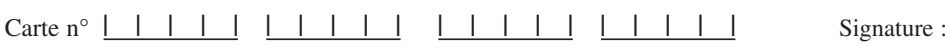

Date d'expiration: $\quad \underline{1}|\underline{1}|$

$\mathrm{N}^{\circ}$ de contrôle au dos de la carte : 